\title{
Microbial interactions between amylolytic and non-amylolytic lactic acid bacteria strains isolated during the fermentation of pozol
}

\author{
Sandra Bolaños-Nuñez ${ }^{1}$, Jorge A. Santiago-Urbina ${ }^{2}$, Jean-Pierre Guyot ${ }^{3}$, Gloria Díaz- Ruiz ${ }^{1}$, Carmen Wacher ${ }^{*}$ \\ 1 Departamento de Alimentos y Biotecnología, Facultad de Química, Universidad Nacional Autónoma de \\ México, Ciudad de México 04510, México; zandybn@gmail.com (S.B.N.); gloriadr@unam.mx (G.D.R.); \\ wacher@unam.mx (C.W.) \\ 2 División de Dirección de Carrera de Agricultura Sustentable y Protegida, Universidad Tecnológica de los \\ Valles Centrales de Oaxaca, Zimatlán 71270, Oaxaca, México; jorgesantiago.urbina@gmail.com \\ 3 Unidad Nutripass, IRD, Montpellier University, 34394 Montpellier, France; nutripass@ird.fr \\ * Correspondence: wacher@unam.mx; Tel.: 5556225315
}

\begin{abstract}
Pozol is a Mexican beverage prepared from fermented nixtamalized maize dough. To contribute to understanding its complex microbial ecology, the effect of inoculating on MRS-starch pure and mixed cultures of amylolytic Sii-25124 and non-amylolytic W. confusa 17, isolated from pozol, were studied on their interactions and fermentation parameters. These were compared with $L$. plantarum A6, an amylolytic strain isolated from cassava. Microbial growth, kinetic parameters, amylolytic activity, lactic acid production, and hydrolysis products from starch fermentation were measured. The population dynamics were followed by qPCR. L. plantarum A6 showed higher enzymatic activity, lactic acid, biomass production, and kinetic parameters than pozol LAB in pure cultures. Mixed culture of each pozol LAB with L. plantarum A6 showed a significant decrease in amylolytic activity, lactic acid yield, specific growth rate, and specific rate of amylase production. The interaction between Sii-25124 and W. confusa 17 increased the global maximum specific growth rate

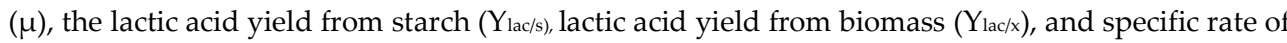
lactic acid production (qlac) by 15, 30, 30, and 40\%, respectively compared with the pure culture of Sii-25124. Interactions between the two strains are essential for this fermentation.
\end{abstract}

Keywords: Pozol, lactic acid bacteria, fermentation, starch, microbial dynamics

\section{Introduction}

Fermented cereals play an essential role in human nutrition in all parts of the world $[1,2]$. In Southeastern México, pozol, a traditional refreshing non-alcoholic beverage based on nixtamalized maize, is consumed [3]. It is a popular beverage that is part of the diet, especially in rural households and village communities. The process production involves cooking maize in an alkaline solution containing $1 \%$ lime $(\mathrm{CaO})$; these cooked corn grains (named nixtamal) are washed with water and milled to make a dough called masa or nixtamal dough. It is shaped into balls, wrapped in banana plant leaves, and left at ambient temperature, usually $30-35$ or even $40^{\circ} \mathrm{C}$, for 3 to 5 hours, one week, one month, or more. It is consumed immediately after preparing it or after fermentation [4]. Pozol is suspended in water and consumed. A natural fermentation, without an intentional inoculum, but knowing that most microorganisms are introduced into the dough during the milling process, occurs [5]. Microbial groups, mainly lactic acid bacteria (LAB), enterobacteria, Bacillus spp., yeasts, and molds, have been detected [3, 6-9]. This process is dominated by LAB, mainly Streptococcus genus [6, 8]. The first day of fermentation is characterized by the presence of amylolytic lactic acid bacteria (ALAB), such as Streptococcus bovis $[8,10]$, identified now as Sii-25124 (Streptococcus infantarius ssp. infantarius); smaller concentrations of Lactobacillus fermentum, Lactobacillus plantarum, Enterococcus, Leuconostoc, 
and Lactococcus $[8,9]$. These LAB are essential microorganisms for pozol fermentation, as most of them can use starch $[8,10]$, the most abundant constituent of maize. Thus, it has been postulated that ALAB plays a vital role in making starch available for other microorganisms [6]. This microbial group contributes to the stability of the product by inhibiting the unwanted microorganisms [11]; they also develop characteristics such as texture and impact in the product's sensory attributes $[12,13]$. Therefore, the selection of starters that traditionally address the fermentation could help to improve the quality of the final product for the benefit of the producers and communities that consume it. To produce pozol under controlled conditions, it would be essential to inoculate those that could direct the fermentation process. These should be able to ferment the substrate effectively. Streptococci and Weissella have been detected in most of the samples that have been analyzed [6, 8]. Pozol fermentation is a complex process, as it is based on a substrate that contains different kinds of compounds and a varied microbiota, which most likely interact.

This study aimed to determine the presence of bacterial interactions between amylolytic and non-amylolytic LAB. We aim to determine if growth in MRS-glucose or MRSstarch, and amylolytic activity of pure and mixed cultures of predominant bacterial species previously isolated from pozol: Sii-25124 (amylolytic) and W. confusa 17 (non-amylolytic), and Lactobacillus plantarum A6, a highly amylolytic strain isolated from cassava (as a control), depending on the use of pure and mixed cultures of these bacteria.

\section{Materials and Methods}

\subsection{Bacterial strains}

Streptococcus infantarius ssp. infantarius 25124, an amylolytic LAB, and Weissella confusa 17, a non-amylolytic LAB isolated from pozol $[8,14]$ were used. In addition, Lactobacillus plantarum A6, previously isolated during cassava retting [15] kindly provided by Dr. Jean-Pierre Guyot (IRD, France), was used as a positive control for amylolytic activity. All strains were maintained in the stock culture collection of the laboratory and kept in 30\% glycerol at $-70^{\circ} \mathrm{C}$.

\subsection{Inoculum preparation}

The strains were reactivated by streaking onto de Man Rogosa and Sharpe (MRS) agar $\left(\mathrm{DB}\right.$ difco $\left.{ }^{\mathrm{tm}}\right)$ and incubated at $30^{\circ} \mathrm{C}$ for $48 \mathrm{~h}$. Then, they were cultivated in $10 \mathrm{~mL}$ of appropriated culture media. W. confusa 17 was inoculated in MRS-glucose broth (MRS-G; BD Difco, Sparks, MD, USA); while Sii-25124 and L. plantarum A6 were grown in modified-MRS-starch (tryptone (10 g/L; BD-Difco, Sparks, MD, USA), ammonium citrate (2.17 g/L; JT Baker, Phillipsburg, NJ, USA), sodium acetate (5 g/L; JT Baker, Phillipsburg, NJ, USA), magnesium sulphate heptahydrate (0.207 g/L; Sigma, St. Louis, MO, USA), manganese sulphate $(0.056 \mathrm{~g} / \mathrm{L}$; Sigma, St. Louis, MO, USA), dipotassium phosphate $(2.62 \mathrm{~g} / \mathrm{L}$; JT Baker, Phillipsburg, NJ, USA), meat extract (10 g/L; BD-Difco, Sparks, MD, USA), yeast extract (5 g/L; BD-Difco, Sparks, MD, USA), and starch (20 g/L; Prolabo, Eurolab). All strains were incubated at $30^{\circ} \mathrm{C}$ for $24 \mathrm{~h}$. For the inoculum preparation, $10 \mathrm{~mL}$ of the previously activated strain were transferred to $100 \mathrm{~mL}$ of MRS-S or MRS-G according to the strain and incubated at $30^{\circ} \mathrm{C}$ overnight. The cultures were used as starters for the following experiments.

\subsection{Monoculture fermentation}

Fermentations of MRS-S broth using pure and mixed cultures were carried out in 1 L Erlenmeyer flasks containing $800 \mathrm{~mL}$ of culture medium at $30^{\circ} \mathrm{C}$ for $24 \mathrm{~h}$, statically. The sterilized medium was inoculated with the pure cultures grown overnight to start at a concentration of $1 \times 10^{6} \mathrm{UFC} / \mathrm{mL}$, corresponding to an optical density of 0.1 at $\mathrm{OD}(600 \mathrm{~nm})$. All experiments were performed in triplicate. 


\subsection{Mixed culture fermentations}

Three different mixed cultures were performed for the assessment of bacterial interactions. The first mixed culture consisted of Sii-25124 and L. plantarum A6, the second mix included Sii-25124 and W. confusa 17, while the third involved L. plantarum A6 and W. confusa 17. Both strains were inoculated simultaneously to MRS-S broth in a ratio of 1:1 to achieve approximately $1 \times 10^{6}$ cells $/ \mathrm{mL}$. Fermentation was performed at $30^{\circ} \mathrm{C}$ for $24 \mathrm{~h}$, statically. All experiments were performed in triplicate.

\subsection{Sample collection}

During the first seven $\mathrm{h}$ of fermentation, samples were collected every $30 \mathrm{~min}$. After that, samples were taken every hour until $12 \mathrm{~h}$ after the fermentation had started, a final sample was collected at $26 \mathrm{~h}$ of fermentation. The $\mathrm{pH}$ and microbial counts of each sample were recorded.

\subsection{Biomass determination}

A calibration curve of optical density at $600 \mathrm{~nm}\left(\mathrm{OD}_{600}\right)$ and cell dry weight was established for each strain. Biomass concentration was determined by measuring the OD 600 with Spectronic 21D spectrophotometer (Milton Roy) and related to dry weight measured after two washing and centrifugation cycles, followed by a drying step at $80^{\circ} \mathrm{C}$ for $24 \mathrm{~h}$. A calibration curve was previously performed between the optical density and the dry weight.

\subsection{Determination of $\alpha$-amylase activity}

For determination of $\alpha$-amylase activity, $10 \mathrm{~mL}$ of the fermented broth were centrifuged at $10000 \mathrm{rpm}, 4{ }^{\circ} \mathrm{C}$ for $15 \mathrm{~min}$. To detect cell-bound amylase activity of Sii-25124, cell pellets were recovered, washed, and suspended in $0.1 \mathrm{M}$ phosphate buffer ( $\mathrm{pH}$ 6.8). Amylase activity was assayed at $\mathrm{pH} 6.8$ and $37^{\circ} \mathrm{C}$ by measuring the iodine-complexing ability of starch as described by Agati et al. [16]. To determine the amylase activity of $L$. plantarum $\mathrm{A} 6,10 \mathrm{~mL}$ of fermented broth was centrifuged at $10000 \mathrm{rpm}, 4^{\circ} \mathrm{C}$ for $15 \mathrm{~min}$, and the cell-free supernatant was recovered as a crude enzyme extract. Enzymatic activity was tested at $\mathrm{pH} 5.0$ and $65^{\circ} \mathrm{C}$ using the starch-iodine extinction method [17]. The lack of amylolytic activity of $W$. confusa 17 , was proved on cells and on the supernatant.

\subsection{HPLC analysis}

Samples were centrifuged at $10000 \mathrm{rpm}$ for $10 \mathrm{~min}$, and supernatants were filtered through a $0.22 \mu \mathrm{m}$ Millipore membrane filter (EMD Millipore, MA, USA). Lactic acid and starch hydrolysis products were determined with the liquid chromatography system (Perkin Elmer 250), equipped with a refractive index detector (Perkin-Elmer 30). Anion-exclusion aminex HPX-87H column ( 300 × $7.8 \mathrm{~mm}$; Bio-Rad, USA) was used. Sulfuric acid (0.01 $\mathrm{N}$ ) was used as the mobile phase at a flow rate of $0.6 \mathrm{~mL} / \mathrm{min}$ and a column temperature of $50^{\circ} \mathrm{C}$. L-Lactate (L-222; Sigma) was used as standard. Analysis of hydrolysis products from starch fermentation was performed using high-performance liquid chromatography equipped with the refractive index detector and a Prodigy 5 ODS 2 C18 column $(250 \times 4.6$ $\mathrm{mm}$; Phenomenex). The products were eluted with water at a flow rate of $0.8 \mathrm{~mL} / \mathrm{min}$ and a column temperature of $35^{\circ} \mathrm{C}$. Glucose, maltose, maltotriose, maltotetraose, maltopentaose, maltohexaose, maltoheptaose were identified by comparing their retention time with standards (Sigma-Aldrich).

\subsection{Sugars quantification}

Total and reducing sugars were determined using the Dubois et al. [18] and Miller methods [19]. 


\subsection{Kinetic parameters}

The maximum specific growth rate, product and growth yields relative to substrate ( $Y_{\text {lacks }}$ and $\mathrm{Y}_{\mathrm{x} / \mathrm{s},}$, respectively), lactic acid and amylase yields from biomass ( $\mathrm{Y}_{\mathrm{lac} / \mathrm{x}}$ and $\mathrm{Y}_{\mathrm{amy} / \mathrm{x}}$, respectively), specific rates of lactic acid or amylase production (qlac and qamy, respectively) and substrate consumption ( $\mathrm{q}_{\mathrm{s}}$ ) were calculated as indicated by Díaz-Ruiz et al. [8].

Significant differences among the different cultures were determined for kinetic parameters by one-way analysis of variance (LSD, $\alpha=0.05$ ). The statistical software Statgraphics Centurion XVI.I.

\subsection{DNA extraction}

DNA from pure and mixed culture was extracted using the MagMax ${ }^{\mathrm{TM}}$ Nucleic Acid isolation kits (Ambion $\left.{ }^{\circledR}\right)$. Twenty milliliters of each culture were centrifuged for $15 \mathrm{~min}$ at $10000 \mathrm{rpm}$ at $4^{\circ} \mathrm{C}$. Pellets were washed three times with sterile distilled water, and each sample was resuspended in $1 \mathrm{~mL}$ of sterile distilled water. Bacterial suspensions were stored at $-21^{\circ} \mathrm{C}$ or directly processed according to the manufacturer's instructions.

\subsection{Real-time PCR primer design and assay conditions}

Primers for detecting phenylalanine transfer RNA (tRNAphe) gene sequences of S.ii 25124, L. plantarum A6, and W. confusa 17 were designed by Applied Biosystems.

Real-time PCR assays were performed on an Applied Biosystems Prism 7500 RealTime PCR System (Applied Biosystemstm). qPCR was realized using HotStart-IT SYBR Green qPCR Master Mix (Thermo Scientific). The $25 \mu \mathrm{L}$ reaction volume consisted of 12.5 $\mu \mathrm{L}$ of $2 X$ HotStart-IT SYBR Green qPCR Master Mix, $0.5 \mu \mathrm{M}$ of forward and reverse primers, and 100 to $0.01 \mathrm{ng}$ of DNA were mixed. PCR conditions were denaturation at $95^{\circ} \mathrm{C}$ for $10 \mathrm{~min}$, followed by 40 cycles of amplification: $15 \mathrm{~s}$ at $95^{\circ} \mathrm{C}, 1 \mathrm{~min}$ at $60^{\circ} \mathrm{C}$, and $72^{\circ} \mathrm{C}$ for $1 \mathrm{~min}$. Fluorescence was detected at the end of the elongation phase for each cycle.

\subsection{Calibration of standards}

Serial dilutions from 100 to $0.1 \mathrm{ng} / \mu \mathrm{L}$ of DNA from single strains samples (Sii-25124, L. plantarum A6, and W. confusa 17 ) were amplified using the primers and the abovementioned conditions. DNA was quantified with a nanodrop spectrophotometer (Agilent 8453). Each reaction was run in triplicate. Amplification efficiency was determined by plotting the threshold cycle for a 10-fold dilution series against the logarithm of the DNA concentration. Efficiency (E) of the qPCR assay was calculated using the equation $E=\left[10^{\wedge}(-\right.$ 1/slope)-1]x100, where the slope refers to the slope of the standard curve for the dilutions series used in qPCR assay. DNA concentration of pure LAB was quantified using the mean values of $\mathrm{CT}$ obtained in three independent assays.

\section{Results}

\subsection{Growth of amylolytic and non-amylolytic-LAB in pure and mixed culture}

The ability of amylolytic and non-amylolytic LAB to grow on MRS-starch broth was evaluated in pure and mixed cultures during the first seven $h$ of fermentation. As pure cultures, Sii-25124 presented a higher growth rate than that of L. plantarum A6 or W. confusa 17 (Figure 1). After this time, these LAB (Sii-25124) remained in stationary phase, producing a maximum biomass concentration of $1.08 \mathrm{~g} / \mathrm{L}$, while that of L. plantarum A6 was $3.8 \mathrm{~g} / \mathrm{L}$ after $24 \mathrm{~h}$ of fermentation. A similar biomass concentration $(3.6 \mathrm{~g} / \mathrm{L})$ was produced with L. plantarum A6 and Sii-25124 co-culture. In the early stages of fermentation, this mixed culture showed the highest growth rate than those achieved by other pure or mixed culture fermentation (Figure 1). From 8 to $12 \mathrm{~h}$ of the experiment, L. plantarum A6 in co-fermentation with $W$. confusa 17 showed higher biomass concentration $(3.2 \mathrm{~g} / \mathrm{L})$ than the co-culture L. plantarum A6-Sii-25124, and Sii-25124-W. confusa 17. 


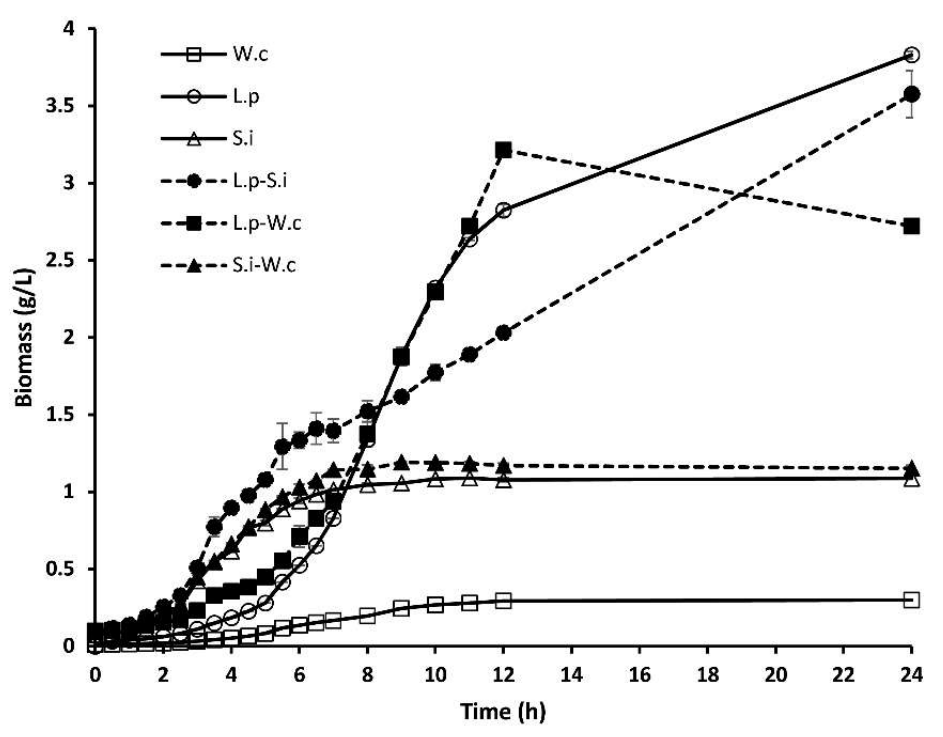

Figure 1. Biomass concentration during fermentation of MRS-starch broth at $30^{\circ} \mathrm{C}$ using pure and mixed cultures of L. plantarum A6, Sii 25124, and W. confusa A17. Results are means of three independent fermentations. W.c: W. confusa 17; L.p: L. plantarum A6; S.i: Sii 25124. Vertical lines show standard deviations.

In the previous experiments, biomass was measured as the dry weight of either single or mixed cultures. To detect the growth dynamics and effects of each of the inoculated LAB during MRS-starch broth fermentation, real-time PCR assay was also employed, using a specific probe for each strain. Findings were in accordance with the information obtained using biomass determination by dry weight. In pure culture, $L$. plantarum A6 reached the highest DNA concentration $(\sim 10,000 \mathrm{ng} / \mathrm{mL})$, followed by Sii25124 (Fig 2A), whereas W. confusa 17 did not grow. As shown in Figure 2B, in mixed fermentation with L. plantarum A6 and Sii-25124, the first species reached $\sim 6000 \mathrm{ng} / \mathrm{mL}$ DNA, and the second one only increased to $\sim 300 \mathrm{ng} / \mathrm{mL}$. Thus, they coexisted, but $L$. plantarum A6 dominated the fermentation. However, in the mixed cultures of W. confusa 17 with L. plantarum A6 or with Sii-25124, W. confusa 17 was excluded from the fermentation, which L. plantarum A6 dominated, that reached $10,500 \mathrm{ng} / \mathrm{mL}$ DNA (Figure 2C), a similar concentration as that obtained as a pure culture. In the mixed culture with Sii25124 and W. confusa 17, starch fermentation was dominated by Sii-25124 (Fig 2D). However, the DNA concentration was only one sixth $(\sim 900 \mathrm{ng} / \mathrm{mL})$ of the concentration reached as a pure culture ( $6000 \mathrm{ng} / \mathrm{mL}$, Fig $2 \mathrm{~A})$. 

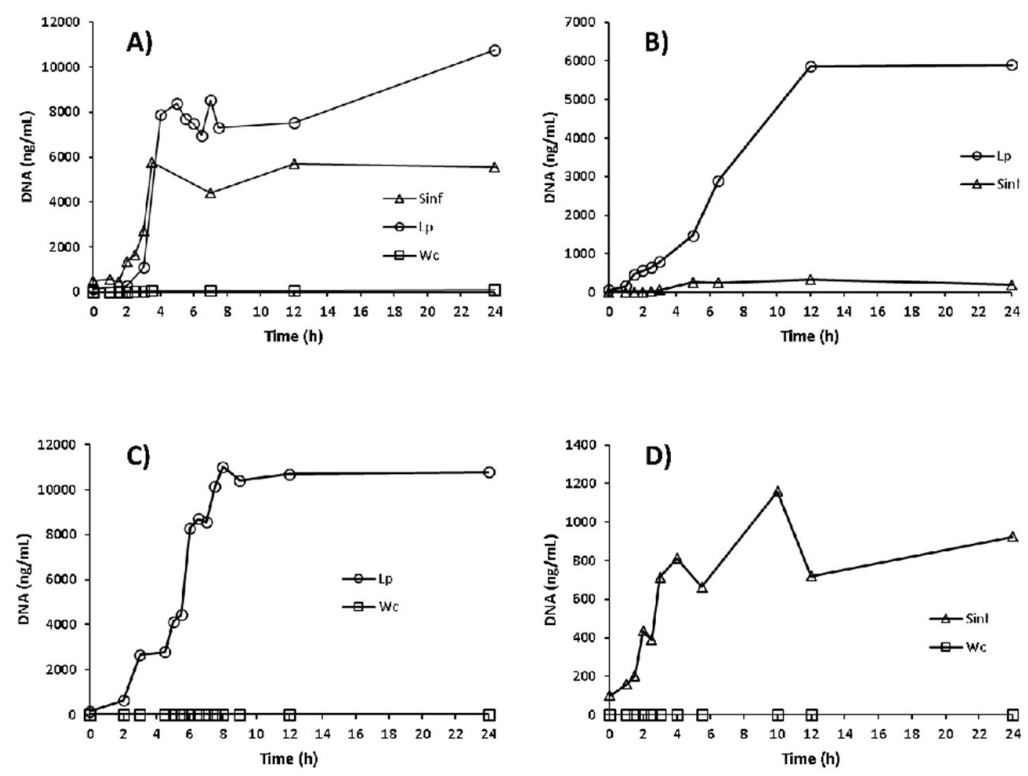

Figure 2. Growth, measured by DNA (Quantitative PCR) of L. plantarum A6 (Lp), Sii. 25124 (Sinf) and W. confusa 17 (Wc) DNA during the fermentation. A) Fermentation performed by pure cultures; B) fermentation performed by L. plantarum A6 and Sii 25124; C) fermentation performed by L. plantarum A6 and W. confusa 17; D) fermentation performed by Sii 25124 and W. confusa 17.

\subsection{Starch hydrolysis}

As expected, Weissella confusa 17 could not hydrolyze starch present in the medium (Figure 3A). However, from 2.5 at six h of fermentation, the mixed culture of L. plantarum A6 and Sii-25124 presented the fastest starch hydrolysis. Whenever L. plantarum A6 was present, the starch was completely hydrolyzed (residual starch below $1 \mathrm{~g} / \mathrm{L}$ ) from 12 $\mathrm{h}$ of the assay. On the other hand, Sii-25124 in monoculture and co-culture with W. confusa 17 could not hydrolyze starch completely (98 and 5.29 g/L of residual starch, respectively).

Reducing sugars were produced in concentrations of 4 to $7 \mathrm{~g} / \mathrm{L}$ (Figure 3C). The maximum sugar concentration was in accordance with the lower starch concentration in the medium from 6 to $12 \mathrm{~h}$ of trial. In the case of W. confusa 17, Sii-25124, and their mixture, reducing sugar concentration did not increase (Figure 3C). 

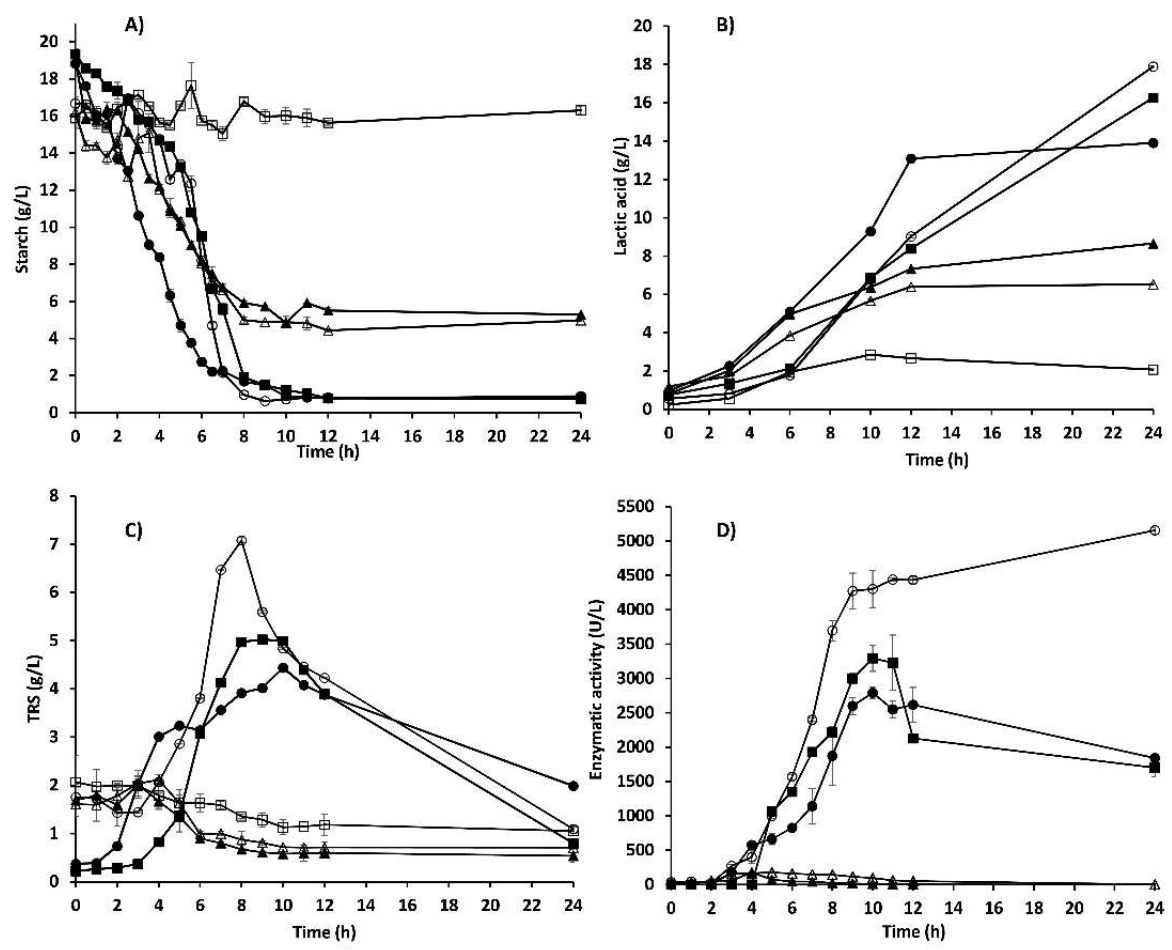

Figure 3. Changes in concentrations of A) starch, B) lactic acid, C) total reducing sugar, and D) enzymatic activity during MRS-starch broth fermentation. L. plantarum A6 (०);

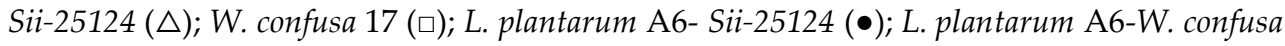
17 (घ); Sii-25124 - W. confusa $17(\boldsymbol{\Delta})$ at $30^{\circ} \mathrm{C}$. Results are means of three independent fermentations.

\subsection{Amylolytic activity and starch hydrolysis products}

In monoculture, Sii-25124 showed low enzymatic activity during the first two hours of fermentation. After five h, this activity reached $176 \mathrm{U} / \mathrm{L}$ (Figure 3D), and then decreased to $1 \mathrm{U} / \mathrm{L}$. Thus, during the first six $\mathrm{h}$ of fermentation, an increase in maltopentaose, maltotriose, and glucose concentration (starch hydrolysis products) were detected (Figure 4A). The amylolytic activity of the single and mixed cultures of L. plantarum A6 was higher than that of other cultures (Figure 3D). At nine h of fermentation, the amylolytic activity reached a value of $4273 \mathrm{U} / \mathrm{L}$; after this time, the activity increased slowly until $5155 \mathrm{U} / \mathrm{L}$ at $24 \mathrm{~h}$ (Figure 3D). These results were in accord with the data obtained from the quantification of maltooligosaccharides. It was found that maltose, maltotriose, and glucose concentrations increased during the third and $12 \mathrm{~h}$ of fermentation (Figure 4B). In the mixed culture of Sii-25124 and W. confusa 17, the maximum enzymatic activity $(176 \mathrm{U} / \mathrm{L})$ was reached at four $\mathrm{h}$ of fermentation. From this point, the amylolytic activity decreased rapidly to $72 \mathrm{U} / \mathrm{L}$. In trials performed using co-culture of $L$. plantarum A6 and W. confusa 17, the amylase production was evident after five h of fermentation. This mixed culture showed a maximum activity (3293 U/L) at ten h of fermentation. This enzymatic activity was higher than that reached by the mixed culture of L. plantarum A6 and Sii-25124 (2791 U/L). The $\alpha$-amylase activity of L. plantarum A6 fermentation was the highest, while $W$. confusa 17 did not show activity. These results agree with the data obtained from the quantification of maltooligosaccharides, where an increase in starch hydrolysis products was not evident, as happened with the cultures of $L$. plantarum A6 or Sii-25124 (Figure 4A, B, C). 

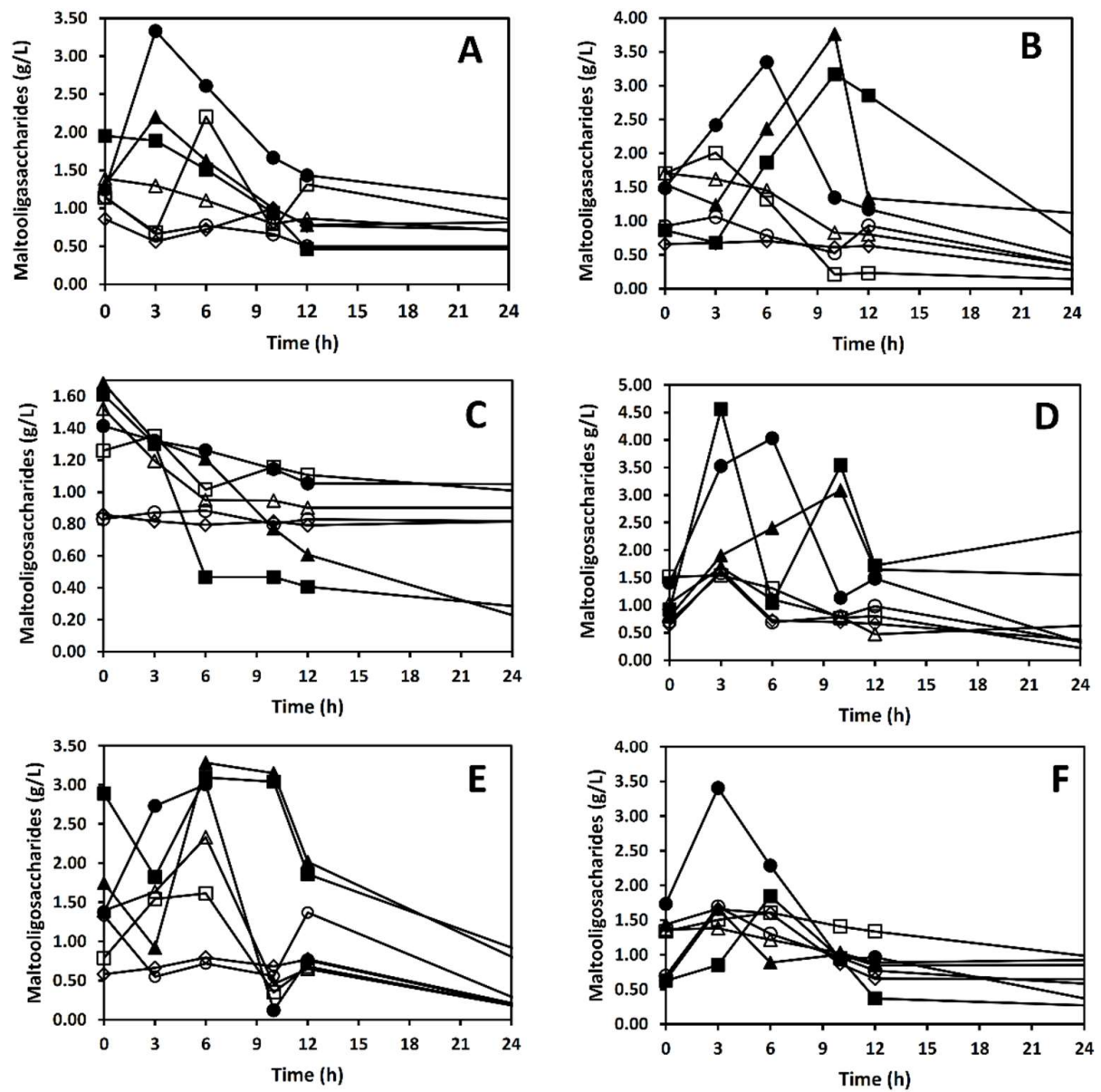

Figure 4. Hydrolysis products from starch fermentation using A) Sii-25124; B) L. plantarum A6; C) W. confusa 17; D) L. plantarum A6- Sii-25124; E) L. plantarum A6-W. confusa 17; F) Sii-25124 -W. confusa 17. Glucose $(\mathbf{\Delta})$, maltose $(\boldsymbol{\bullet})$, maltotriose $(\bullet)$, maltotetraose $(\triangle)$, maltopentaose $(\square)$, maltohexaose $(\circ)$, maltoheptaose $(\diamond)$.

\subsection{Lactic acid production}

The maximum lactic acid concentrations produced by L. plantarum A6, Sii-25124, and $W$. confusa 17 in pure culture were 17.90, 6.5, and $2 \mathrm{~g} / \mathrm{L}$, respectively. At 10 and $12 \mathrm{~h}$ of the assay, L. plantarum A6 in co-culture with Sii-25124 produced the highest lactic acid concentration (Figure 3B). However, in the co-cultures of L. plantarum A6 and Sii-25124 or W. confusa 17, the lactic acid concentration was lower than that reached as in pure culture, it was $17.90 \mathrm{~g} / \mathrm{L}$ to 16 and $14 \mathrm{~g} / \mathrm{L}$, respectively (Figure 3B). On the contrary, in the co-culture of Sii-25124 and W. confusa 17, the lactic acid concentration increased to $8 \mathrm{~g} / \mathrm{L}$, compared to $6.5 \mathrm{~g} / \mathrm{L}$ produced by Sii-25124 (Figure 3B).

\subsection{Efficiency of fermentation}

As shown in Table 1, the highest lactic acid yield (Ylac/s) was produced by L. plantarum A6 (1.09 g/g), and the lowest Ylac/s was obtained from the W. confusa $17(0.32 \mathrm{~g} / \mathrm{g})$.

Mixed cultures containing L. plantarum A6 reached Ylac/s $0.8 \mathrm{~g} / \mathrm{g}$. In general, mono and cocultures including L. plantarum A6 showed high values for biomass/substrate yield, $\alpha$ amylase/biomass yield, $\alpha$-amylase/substrate yield, and qamy compared to W. confusa 
17 and Sii-25124. In addition, the maximum specific growth rate $(\mu)$ was higher for L. plantarum A6 than Sii-25124 and W. confusa 17 (Table 1). However, when W. confusa 17 and Sii-25124 were mixed, the $\mu$ value increased 18\% (Table 1), compared to Sii-25124 as pure cultures.

Table 1. Kinetic parameters of starch fermentation by pure and mixed culture of LAB

\begin{tabular}{|c|c|c|c|c|c|c|c|c|c|}
\hline \multirow{3}{*}{ Inoculum } & \multicolumn{9}{|c|}{ Parameters } \\
\hline & $Y_{x / s}$ & Ylac/s & Ylac/x & $Y_{a m y / x}$ & Yamy/s & $\mu$ & qlac $^{a}$ & $\mathrm{qs}^{\mathrm{b}}$ & $\mathrm{qamy}^{\mathrm{c}}$ \\
\hline & $\mathrm{g} / \mathrm{g}$ & $\mathrm{g} / \mathrm{g}$ & $\mathrm{g} / \mathrm{g}$ & $\mathrm{U} / \mathrm{g}$ & $\mathrm{U} / \mathrm{g}$ & $h^{-1}$ & & & \\
\hline L. plantarum & $0.24 \pm 0.03^{\mathrm{d}}$ & $1.09 \pm 0.13^{\mathrm{e}}$ & $4.57 \pm 0.02^{\mathrm{b}}$ & $1348.53 \pm 20.40^{c}$ & $323.39 \pm 40.82^{\mathrm{bc}}$ & $0.43 \pm 0.00^{\mathrm{d}}$ & $1.95 \pm 0.01^{c}$ & $1.79 \pm 0.20^{\mathrm{b}}$ & $577.37 \pm 8.74^{\mathrm{d}}$ \\
\hline S. infantarius & $0.09 \pm 0.00^{\mathrm{b}}$ & $0.50 \pm 0.00^{\mathrm{b}}$ & $5.27 \pm 0.03^{c}$ & $200.74 \pm 3.15^{b}$ & $18.73 \pm 0.50^{\mathrm{a}}$ & $0.28 \pm 0.02^{\mathrm{b}}$ & $1.49 \pm 0.07^{b}$ & $2.96 \pm 0.04^{\mathrm{d}}$ & $56.75 \pm 0.89^{a}$ \\
\hline W. confusa & $0.027 \pm 0.00^{a}$ & $0.32 \pm 0.02^{\mathrm{a}}$ & $10.21 \pm 0.05^{\mathrm{f}}$ & nd & nd & $0.27 \pm 0.01^{\mathrm{b}}$ & $2.71 \pm 0.13^{e}$ & $9.70 \pm 0.16^{\mathrm{e}}$ & nd \\
\hline L.p-S.i & $0.22 \pm 0.01^{\mathrm{d}}$ & $0.82 \pm 0.00^{\mathrm{cd}}$ & $3.73 \pm 0.16^{\mathrm{a}}$ & $1668.66 \pm 2.11^{\mathrm{d}}$ & $334.21 \pm 1.05^{c}$ & $0.11 \pm 0.01^{\underline{a}}$ & $0.4 \pm 0.02^{\underline{a}}$ & $0.48 \pm 0.02^{a}$ & $177.89 \pm 0.22^{\mathrm{b}}$ \\
\hline L.p-W.c & $0.15 \pm 0.00^{c}$ & $0.86 \pm 0.02^{\mathrm{d}}$ & $5.91 \pm 0.11^{\mathrm{d}}$ & $1232.33 \pm 173.22 \mathrm{c}$ & $280.71 \pm 14.62^{\mathrm{b}}$ & $0.33 \pm 0.01^{c}$ & $1.94 \pm 0.03^{c}$ & $2.26 \pm 0.02^{c}$ & $404.00 \pm 56.85^{c}$ \\
\hline S.i-W.c & $0.11 \pm 0.00^{\mathrm{b}}$ & $0.72 \pm 0.02^{c}$ & $7.43 \pm 0.02^{\mathrm{e}}$ & $303.85 \pm 16.80^{\mathrm{b}}$ & $27.16 \pm 1.34^{\mathrm{a}}$ & $0.33 \pm 0.01^{c}$ & $2.47 \pm 0.08^{\mathrm{d}}$ & $2.90 \pm 0.14^{\mathrm{d}}$ & $101.03 \pm 4.86^{a}$ \\
\hline
\end{tabular}

L.p: Lactobacillus plantarum A6; S.i: S. infantarius 25124; W.c: Weisella confusa. Biomass yield $\left(\mathrm{Y}_{\mathrm{X} / \mathrm{s}}\right)$ was calculated as grams of biomass produced per gram of utilized sugar; lactic acid yield (Ylacs) was calculated as grams of lactic acid produced per gram of utilized sugar; lactic acid yield (Ylack $x$ ) was calculated as grams of lactic acid produced per gram of dry cell weight; amylase yield (Yamy/x) was calculated as units of amylase produced per gram of dry cell weight; amylase yield (Yamy/s) was calculated as units of amylase produced per gram of utilized sugar; $\mu$ : maximum specific growth rate; qac: specific rate of lactic acid production; qs: specific rate of substrate consumption; qamy: specific rate of amylase production. a: $\mathrm{g}$ of lactic acid/g of cell dry wt*h; $\mathrm{b}$ : $\mathrm{g}$ of substrate/g of cell dry $\mathrm{wt} t^{*} \mathrm{~h} ; \mathrm{c}: \mathrm{U} / \mathrm{g}$ of cell dry wt*h. nd: non-determined. These values are the mean \pm standard deviation of three experiments. Different lowercase letters in the same column show significant differences according to the analysis of variance at $\mathrm{p} \leq 0.05$ (LSD test). 


\section{Discussion}

In pozol fermentation, starch from nixtamal has been reported to be the main carbon and energy source available to develop the microbiota [10, 8]. Both amylolytic and non-amylolytic lactic acid bacteria have been identified [10, 8]. It has been reported that after six $h$ pozol fermentation, the concentration of non-amylolytic LAB is higher than that of ALAB [8] then ALAB should allow the growth of non-amylolytic LAB, as a result of starch degradation and maltodextrin and maltose production [10]. In the present study, the effect of these bacteria in pure and mixed cultures on fermentation efficiency, $\alpha$-amylase activity, dynamics, and microbial growth of pure and mixed cultures of amylolytic LAB and non-amylolytic LAB on MRS-starch broth were studied. MRS-starch broth was used because of the easiness of handling this as a model of starch fermentation instead of pozol nixtamal dough.

Results showed that the amylolytic strain L. plantarum A6 produced more biomass than Sii-25124 and W. confusa. L. plantarum A6 is known as a highly amylolytic strain that has been used in different processes to produce pearl milled soybean fermented gruels [20], pearl milled-porridge [21], and sorghum porridge [22]. In this work, $L$. plantarum A6 showed higher microbial growth and $\alpha$-amylase activity than Sii-25124 and $W$. confusa. It is probably due to extracellular alpha-amylase and neopullulanase activities [21, 23] and the presence of maltodextrin transporters in L. plantarum [23]. Also, amylopectin phosphorylase and alpha-glucosidase enzymes that could be involved in starch hydrolysis [21]. This is possibly why the mixed cultures that included L. plantarum A6 showed higher values of kinetic parameters of starch fermentation (Table 1), $\alpha$-amylase activity, and microbial biomass (Figure 3D and Figure 1) than the rest of the cultures. On the other hand, Sii-25124 growth was lower than that of L. plantarum A6. Therefore, it could be associated with the culture medium composition and bacteria adaptation. Sii25124 also is adapted to metabolize xylan and arabinoxylan, the second-largest fermentable carbohydrate in nixtamal dough, contributing greatly to their predominance in pozol [24]. However, its initial growth rate was the highest, and this is an important trait, as the antimicrobial activity would be present earlier than that of the other strains. This is an important issue, as it could result in better food safety quality. In the same way, W. confusa 17, being non-amylolytic, did not grow as a pure culture, but neither in the mixed cultures with the two amylolytic strains. Indicating that it does not depend entirely on the amylolytic activity of the ALABs. It has been reported that $W$. confusa can grow using xylan and xylooligosaccharides as a carbon source, predominant carbohydrates in nixtamal dough [25]. This could be one reason for its predominance in pozol dough. However, the media did not contain xylan or xylooligosaccharides.

W. confusa 17 did not present growth nor amylolytic activity. However, the presence of this LAB in mixed culture with Sii-25124 resulted in the increase of Ylac/s and qamy values (Table 1) compared to A6 pure culture. This observation suggests that there is an interaction between both microbial populations when they coexist. Thus, lactic acid production from the mixed culture of these pozol LAB was higher than the one reached as monoculture. Humblot et al. [21] suggested that the expression of amylase genes varies depending on the starchy matrix. So, the enzymatic activity of these pozol strains could be higher in the dough than in MRS-starch broth. In this case, starch was not consumed completely, leaving approximately $30 \%$ of residual starch concentration, similarly to its concentration in pozol [10].

The amylolytic activity of L. plantarum A6 was higher than that of pozol LAB, possibly due to the higher biomass production in the medium. However, when this strain was inoculated in mixed culture with pozol LAB, its amylolytic activity, values of Ylac/s (from $1.09 \mathrm{~g} / \mathrm{g}$ to $0.82 \mathrm{~g} / \mathrm{g}$ ), qamy (from 577.37 to 177.89 ), and $\mu$ (from $0.43 \mathrm{~h}^{-1}$ to $0.11 \mathrm{~h}^{-1}$ ) decreased (Table 1). Diaz-Ruíz et al. [8] suggested that the predominance of the non- 
amylolytic LAB in pozol fermentation was due to the amylolytic activity of ALAB, which releases sugars. The results obtained in this study showed that Weissella confusa 17 grew neither in MRS-starch broth nor in the same medium in co-culture with each of the amylolytic strains, and this suggests it does not depend on the sugars released by these bacteria. As W. confusa 17 does not grow in the medium, it is an interesting result or becomes non-culturable. Instead, it depends, most likely, on other carbohydrates present in pozol dough, such as those reported by Cooper-Bribiesca et al. [24] and López-Hernández et al. [25]. Both authors have found that Sii-25124 and W. confusa 17 can metabolize polysaccharides derived from hemicellulose, a carbohydrate present in the maize pericarp.

Single cultures kinetic data of Sii-25124, L. plantarum A6 y W. confusa 17 were different than those obtained with their co-cultures. Results indicate interactions among them; however, it is still unknown what kind these interactions are.

In combination with L. plantarum A6, both Sii-25124 and W. confusa 17 had a negative influence on the amylolytic activity, lactic acid yield (Ylac/s), specific growth rate $(\mu)$, and specific rate of amylase production (qamy) in the fermentation. Nevertheless, RT-PCR analysis showed that when $W$. confusa 17 was co-inoculated with the other two ALAB, it does not significantly increase its growth. These results suggest that the growth of the non-ALAB in pozol, does not entirely depend on the amylase activity of the ALABs.

\section{Conclusions}

Clear differences in growth and fermentation parameters were found among pure and mixed cultures of the three lactic acid bacteria. L. plantarum A6 dominated in all cases, and W. confusa 17 did not grow. Sii-25124 dominated in co-culture with W. confusa 17 in MRS-starch broth. L. plantarum A6 fermentation parameters were higher than those of pozol LAB; however, growth rate of Sii-25124 in the first hours of fermentation was higher than that of the other bacteria. The advantages of growing in mixed cultures were: In L. plantarum A6-Sii-25124 mixed culture fermentation, the initial growth rate was higher than L. plantarum in pure culture, although biomass was lower. While $W$. confusa 17 affected the growth of L. plantarum A6, as more biomass was produced in its presence. Then, there are interactions among the strains studied, and the nature of these need to be investigated. Starch is essential for nixtamal fermentation, but it is possible that it is not the only or the most important.

This work contributes to the understanding of the complex microbial ecology of pozol.

Author Contributions: Conceptualization, C.W. and S.B.-N.; methodology, C.W.; S.B.-N. and G.D.R.; formal analysis, C.W.; S.B.-N. and J.P.G.; investigation, S.B.-N.; writing - original draft preparation, C.W.; S.B.-N. and J.A.S.-U; writing-review and editing, C.W.; S.B.-N.; J.A.S.-U.; J.P.G and G.D.-R. All authors have read and agreed to the published version of the manuscript.

Funding: This research was funded by CONACyT (National Science and Technology Council) project CB-2009-131615; PAPIIT/UNAM (Programa de Apoyo a Proyectos de Investigación e Innovación Tecnológica, UNAM Universidad Nacional Autónoma de México) project IN223917 and CONACyT (National Science and Technology Council) PhD grant.

Conflicts of Interest: The authors declare no conflict of interest.

\section{References}

1. Brandt, M.J. Starter cultures for cereal based foods. Food Microbiol. 2014, 37, 41-43. doi.org/10.1016/j.fm.2013.06.007 
2. Marsh, A.J.; Hill, C.; Ross, R.P.; Cotter, P.D. Fermented beverages with health-promoting potential: Past and future perspectives. Trends Food Sci. Tech. 2014, 38,113-124. doi.org/10.1016/j.tifs.2014.05.002

3. Wacher, C.; Cañas, A.; Bárzana, E.; Lappe, P.; Ulloa, M.; Owens, J.D. Microbiology of Indian and Mestizo pozol fermentations. Food Microbiol. 2000, 17, 251-256. doi.org/10.1006/fmic.1999.0310

4. Cañas, A.; Barzana, E.; Owens, J.D.; Wacher, C. La elaboración de pozol en los altos de Chiapas. Ciencia 1993, 44, 219-229

5. Wacher, C. Nixtamalization, a Mesoamerican technology to process maize at small-scale with great potential for improving the nutritional quality of maize based foods. In: Food-Based Approaches for a Healthy Nutrition 2003, 735-743.

6. Ampe, F.; Omar, N.B.; Moizan, C.; Wacher, C.; Guyot, J.P. Polyphasic study of the spatial distribution of microorganisms in Mexican Pozol, a fermented maize dough, demonstrates the need for cultivation-independent methods to investigate traditional fermentations. Appl. Environ. Microb. 1999, 65, 5464-5473. doi: 10.1128/AEM.65.12.5464-5473.1999

7. Cárdenas, C.; Barkla, B.J.; Wacher, C.; Delgado-Olivares, L.; Rodríguez-Sanoja, R. Protein extraction method for the proteomic study of a Mexican traditional fermented starchy food. J. Proteomics 2014, 111, $139-147$. doi.org/10.1016/j.jprot.2014.06.028

8. Díaz-Ruiz, G.; Guyot, J.P.; Ruiz-Terán, F.; Morlon-Guyot, J.; Wacher, C. Microbial and physiological characterization of weakly amylolytic but fast-growing lactic acid bacteria: a functional role in supporting microbial diversity in pozol, a Mexican fermented maize beverage. Appl. Environ. Microb. 2003, 69, 4367-4374. doi: 10.1128/AEM.69.8.4367-4374.2003

9. Escalante, A.: Wacher, C.: Farrés, A. Lactic acid bacterial diversity in the traditional Mexican fermented dough pozol as determined by $16 S$ rDNA sequence analysis. Int. J. Food Microbiol. 2001, 64, 21-31. doi: 10.1016/s0168-1605(00)00428-1

10. Ben Omar, N.; Ampe, F. Microbial community dynamics during production of the Mexican fermented maize dough pozol. Appl. Environ. Microb. 2000, 66, 3664-3673. doi: 10.1128/aem.66.9.3664-3673.2000

11. Settanni, L.; Corsetti, A. Application of bacteriocins in vegetable food bio-preservation. Int. J. Food Microbiol. 2008, 121, 123138. doi:10.1016/j.ijfoodmicro.2007.09.001

12. Londoño-Zapata, AF.; Durango-Zuleta, M.M.: Sepúlveda-Valencia, J.U.; Moreno-Herrea, C.X. Characterization of lactic acid bacterial communities associated with a traditional Colombian cheese: Double cream cheese. LWT Food Sci. Technol. 2017, 82, 39-48. doi.org/10.1016/j.lwt.2017.03.058

13. Panda, S.H.; Ray, R.C. Amylolytic lactic acid bacteria Microbiology and technological interventions in food fermentations. Fermented Foods Part I: Biochemistry and Biotechnology, 1st Ed.; Montet, D., Ray, R.C. eds.; Taylor and Francis Group, New York, 2016, pp. 148-165.

14. Flores-Espinosa, M.T. Caracterización fisiológica de las bacterias lácticas aisladas del pozol. Bachelor's thesis, Universidad Nacional Autónoma de México, Mexico City, Mexico, 1996.

15. Giraud, E.; Brauman, A.; Keleke, S.; Lelong, B.; Raimbault, M. Isolation and physiological study of an amylolytic strain of Lactobacillus plantarum. Appl. Microbiol. Biotechnol. 1991, 36, 379-383. doi.org/10.1007/BF00208160

16. Agati, V.; Guyot, J.P.; Morlon-Guyot, J.; Talamod, P.; Hounhounigan, D.J. Isolation and characterization of new amylolytic strains of Lactobacillus fermentum from fermented maize doughs (mawê and ogi) from Benin. J. Appl. Microbiol. 2002, 85, 512-520. doi.org/10.1046/j.1365-2672.1998.853527.x

17. Giraud, E.; Gosselin, L.; Raimbault, M. Production of a Lactobacillus plantarum starter with linamarase and amylase activities for cassava fermentation. J. Sci. Food Agric. 1993, 62, 77-82. doi.org/10.1002/jsfa.2740620111

18. Dubois, M.; Gilles, K.A.; Hamilton, J.K.; Rebers, P.A.; Smith, F. Colorimetric method for determination of sugars and related substances. Anal. Chem. 1956, 28, 350-356. doi.org/10.1021/ac60111a017

19. Miller, G.L. Use of dinitrosalicylic acid reagent for determination of reducing sugars. Anal. Chem. 1959, 31, 426-428. doi.org/10.1021/ac60147a030

20. Songré-Ouattara, L.T.; Mouquet-Rivier, C.; Humblot, C.; Rochette, I.; Diawara, B,.; Guyot, J.P. Ability of selected lactic acid bacteria to ferment a pearl millet-soybean slurry to produce gruels for complementary foods for young children. J. Food Sci. 2010, 75, 261-269. doi:10.1111/j.1750-3841.2010.01640.x

21. Humblot, C.; Turpin, W.; Chevalier, F.; Picq, C.; Rochette, I.; Guyot, J.P. Determination of expression and activity of genes involved in starch metabolism in Lactobacillus plantarum A6 during fermentation of a cereal-based gruel. Int. J. Food Microbiol. 2014, 185, 103-111. doi:10.1016/j.ijfoodmicro.2014.05.016

22. Mukisa, I.M.; Byaruhanga, Y.B.; Muyanja, C.M.; Aijuka. M.; Schüller, R.B.; Sahlstrøm, S.; Langsrud, T.; Narvhus, J.A. Influence of cofermentation by amylolytic Lactobacillus plantarum and Lactococcus lactis strains on the fermentation process and rheology of sorghum porridge. Appl. Environ. Microb. 2012, 78, 5220-5228. doi.org/10.1128/AEM.00857-12

23. Turpin, W.; Weiman, M.; Guyot, J.P.; Lajus, A.; Cruveiller, S.; Humblot, C. The genomic and transcriptomic basis of the potential of Lactobacillus plantarum A6 to improve the nutritional quality of a cereal based fermented food. Int. J. Food Microbiol. 2018, 266, 346-354. doi: 10.1016/j.ijfoodmicro.2017.10.011

24. Cooper-Bribiesca, B.; Navarro-Ocaña, A.; Díaz-Ruiz, G.; Aguilar-Osorio, G.; Rodríguez-Sanoja, R.; Wacher, C. Lactic acid fermentation of arabinoxylan from nejayote by Streptococcus infantarius ssp. Infantarius 25124 from pozol. Front. Microbiol. 2018, 9, 3061. doi: 10.3389/fmicb.2018.03061

25. López-Hernández, M.; Rodríguez-Alegría, M.E.; López-Munguía, A.; Wacher, C. Evaluation of xylan as carbon source for Weisella spp., a predominant strain in pozol fermentation. LWT Food Sci. Technol. 2018, 89, $192-197$. doi.org/10.1016/j.lwt.2017.10.030 